

REVISTA DE LA FACULTAD

DE CIENCIAS DE LA SALUD

\title{
Hallazgos histopatológicos y malignidad de masas suprarrenales en un centro de patología y citología en Bucaramanga, Santander entre 2007 y 2019
}

Histopathological findings and malignancy of adrenal masses in a pathology and cytology center in Bucaramanga, Santander between 2007 and 2019

Achados histopatológicos e malignidade de massas adrenais em um centro de patologia e citologia em Bucaramanga, Santander entre 2007 e 2019

Gustavo Adolfo Parra-Serrano, MD. Esp. ${ }^{1}$ (D), María Paula Torres-Langhammer, Est. ${ }^{2}$ (D), Ana María Castillo-Díaz, MD. ${ }^{3}$ (D), María Emma García-Ardila, MD. Esp. ${ }^{4}$ (D), Sergio Serrano-Gómez, MD. MSc. ${ }^{5}$ iD

1. Médico, Especialista en Medicina Interna y Endocrinología. Fundación Oftalmológica de Santander, Universidad Autónoma de Bucaramanga, Bucaramanga, Colombia.

2. Estudiante de Medicina. Universidad Autónoma de Bucaramanga, Bucaramanga, Colombia.

3. Médica. Universidad del Rosario, Bogotá, Colombia.

4. Médica, Patóloga. Mega Patología y Citología, Bucaramanga, Colombia.

5. Médico, Magíster en Epidemiología. Universidad Autónoma de Bucaramanga, Bucaramanga, Colombia.

Correspondencia. Gustavo Adolfo Parra-Serrano. Calle 158 \# 20 -55 consultorio 408 Floridablanca, Santander. Teléfonos: 3112306707 Email: gparra_serrano@hotmail.com

\section{INFORMACIÓN DEL ARTÍCULO:}

Artículo recibido: 10 de diciembre de 2020

Artículo aceptado: 08 de junio de 2021

DOI: https://doi.org/10.29375/01237047.4054

Cómo citar. Parra-Serrano GA, Torres-Langhammer MP, Castillo-Díaz AM, García-Ardila ME, Serrano-Gómez S. Hallazgos histopatológicos y malignidad de masas suprarrenales en un centro de patología y citología en Bucaramanga, Santander entre 2007 y 2019. MedUNAB. 2021;24(2): 155-161 doi: https://doi.org/10.29375/01237047.4054

RESUMEN:

Introducción. Las masas encontradas en la glándula suprarrenal pueden clasificarse de acuerdo con su origen, comportamiento, localización, función y forma de diagnóstico. En Colombia no existen datos suficientes que describan la frecuencia y las principales características histopatológicas de dichas lesiones. El objetivo del presente manuscrito es describir los principales hallazgos histopatológicos 
y la malignidad de las masas suprarrenales en un centro médico especializado en patología de Bucaramanga, Santander. Metodología. Estudio descriptivo y retrospectivo. Se revisaron patologías de pacientes de todas las edades con alteraciones histopatológicas en la glándula suprarrenal, en un centro médico de Bucaramanga, Santander. Resultados. Se revisaron 79 patologías suprarrenales de las cuales 39 presentaron lesión a nivel de la glándula suprarrenal, la lesión más frecuente encontrada fue la metástasis (28.2\%), y la localización de lesión más frecuente se evidenció en la glándula derecha (62.1\%). Conclusión. Es fundamental que se realicen estudios prospectivos que permitan obtener datos epidemiológicos con el fin de generar datos locales.

Palabras clave:

Malignidad; Patología; Glándulas Suprarrenales; Neoplasia; Metástasis de Neoplasia.

\begin{abstract}
Introduction. Masses found in the adrenal gland can be classified according to their origin, behavior, location, function and manner of diagnosis. In Colombia there are insufficient data describing the frequency and main histopathological characteristics of these lesions. The aim of this manuscript is to describe the main histopathological findings and malignancy of adrenal masses in a medical center specialized in pathology in Bucaramanga, Santander. Methodology. Descriptive and retrospective study. Pathologies of patients of all ages with histopathologic alterations in the adrenal gland were reviewed in a medical center in Bucaramanga, Santander. Results. Seventy-nine adrenal pathologies were reviewed, of which 39 showed adrenal gland lesions. The most frequent lesion found was metastasis (28.2 \%), and the most frequent location of the lesion was in the right gland (62.1\%). Conclusion. It is essential that prospective studies be carried out to obtain epidemiological data in order to generate local data..
\end{abstract}

Keywords:

Malignancy; Pathology; Adrenal Glands; Neoplasm; Neoplasm Metastasis.

\title{
RESUMO
}

Introdução. As massas encontradas na glândula adrenal podem ser classificadas de acordo com a sua origem, comportamento, localização, função e forma de diagnóstico. Na Colômbia, não há dados suficientes que descrevam a frequência e as principais características histopatológicas dessas lesões. O objetivo deste artigo é descrever os principais achados histopatológicos e a malignidade das massas adrenais em um centro médico especializado em patologia em Bucaramanga, Santander. Metodologia. Estudo descritivo e retrospectivo. Foram analisadas patologias de pacientes de todas as idades com alterações histopatológicas na glândula adrenal, em um centro médico em Bucaramanga, Santander. Resultados. Foram revisadas 79 patologias adrenais, das quais 39 apresentavam lesão em glândula adrenal, a lesão mais frequente encontrada foi metástase (28.2\%), e o local mais frequente de lesão foi evidenciado na glândula direita (62.1\%). Conclusão. É imprescindível a realização de estudos prospectivos para obtenção de dados epidemiológicos a fim de gerar dados locais..

Palavras-chave:

Malignidade; Patologia; Glândulas adrenais; Neoplasia; Metástase de neoplasia.

\section{Introducción}

Laglándula suprarrenal es un órgano endocrino localizado en el retroperitoneo, cuenta con dos componentes principales: médula y corteza, el primero se encarga de la síntesis de catecolaminas y el segundo de la producción de mineralocorticoides, glucocorticoides y andrógenos (1). Las masas suprarrenales han incrementado su incidencia y prevalencia entre los últimos 15 y 20 años, probablemente secundario al desarrollo de técnicas imagenológicas más avanzadas y el uso frecuente de las mismas, que permiten su visualización temprana (2-4). Es de gran importancia conocer la etiología, frecuencia, localización y malignización de las mismas para así facilitar su diagnóstico y tratamiento.

Las masas adrenales pueden clasificarse según su origen, comportamiento, localización, función y forma de diagnóstico. En la mayoría de los casos se encuentran de manera incidental, por lo que reciben el nombre de 
incidentalomas; definidas como masas adrenales mayores a un centímetro y caracterizadas por ser encontradas durante la realización de estudios imagenológicos en patologías de etiología no adrenal $(1,2,5)$.

Esimportantedestacarquelafrecuenciadeincidentalomas aumenta con la edad, es extraño su hallazgo en menores de 30 años, y alcanza una frecuencia de hasta el 7\% en adultos mayores de 50 años $(1,3,6)$. Además de esto, se relaciona con patologías como la obesidad, diabetes mellitus e hipertensión arterial $(7,8)$.

Se cuenta con epidemiología a nivel internacional, entre los cuales se encuentran las guías de la Sociedad Europea de Endocrinología del 2016, donde establecen la prevalencia histopatológica de las masas suprarrenales tipo incidentalomas. Se evidencia que la masa más frecuente es el adenoma no funcional hasta en un $80 \%$ de los casos, seguidos por adenomas funcionantes, siendo el más frecuente el secretor de cortisol hasta en un $12 \%$. Adicionalmente, se encuentra el feocromocitoma $7 \%$, carcinoma adrenocortical $8 \%$ y lesiones metastásicas 5\% (9-11). A su vez, un estudio transversal realizado en un hospital de Brasil encontró que la prevalencia de las masas suprarrenales era de un $2.5 \%$, siendo más frecuente en hombres que en mujeres y con un aumento significativo de la prevalencia en edades avanzadas (12) y encontradas de manera unilateral en la mayoría de los casos, lo que concuerda con las guías clínicas argentinas de la evaluación diagnóstica y terapéutica de las masas suprarrenales (8).

En Colombia y Latinoamérica existen pocos estudios que describan la distribución global de la etiología de las masas suprarrenales, las características histopatológicas y la relación con malignidad, lo que limita su diagnóstico y abordaje clínico eficaz. Teniendo en cuenta el impacto clínico y el aumento franco en la incidencia de estas masas, se considera importante describir los principales hallazgos histopatológicos y de malignidad de las masas suprarrenales; determinando la frecuencia de las masas de acuerdo con múltiples variables tales como el sexo y los diferentes grupos etarios. Es fundamental iniciar y fomentar el estudio de las masas suprarrenales en la población colombiana con el fin de obtener epidemiología local, lo que permitirá mejorar el diagnóstico, tratamiento y pronóstico de las mismas.

\section{Metodología}

Se llevó a cabo un estudio descriptivo y retrospectivo en el cual se revisaron los estudios patológicos de pacientes de todas las edades con patologías de la glándula suprarrenal realizadas en el centro Mega Patología y Citología de Bucaramanga entre 2007 y 2019. Los casos fueron identificados por medio de una búsqueda realizada en la base de datos de la institución usando el término "glándula suprarrenal". Posterior a la revisión de las patologías se incluyeron dentro del estudio un total de 79 patologías, de las cuales 40 no mostraban alteraciones y 39 mostraban cambios patológicos de la glándula suprarrenal. Se elaboró una base de recolección de datos, en la que se tuvo en cuenta el diagnóstico realizado por el patólogo, tamaño y peso de la glándula, localización de la lesión, malignidad, y si esta era de origen primario o metastásico; del mismo modo, fueron tomadas en cuenta variables sociodemográficas como el sexo y la edad. La recolección de los datos obtenidos por medio de las patologías se realizó en una base de datos de Excel y posteriormente fueron agrupadas las variables de edad (mayor o menor de 65) y el largo de la masa (mayor o menor a $4 \mathrm{~cm}$ ). El análisis estadístico se realizó en Stata 15.0. Para las medidas cuantitativas se utilizaron medidas de tendencia central y dispersión, y las medidas cualitativas se describieron mediante frecuencias absolutas y relativas. Posteriormente, se realizó un análisis bivariado para determinar si existen asociaciones entre malignidad y otras variables.

\section{Resultados}

Para el estudio fueron utilizadas 79 patologías de glándula suprarrenal, recolectadas desde enero del 2007 hasta octubre del 2019, 39 (49.3\%) de estas reportaron hallazgos histopatológicos y $40 \quad(50.7 \%)$ fueron reportadas como normales. Los hombres representaron el $62.3 \%$ de las patologías realizadas en la institución. El resto de los resultados fueron analizados teniendo en cuenta únicamente las 39 patologías que tuvieron hallazgos histopatológicos. Con respecto a la edad, se evidenció que solo el $19.5 \%$ eran pacientes mayores de 65 años, y la media de edad fue de 55.2 años, con un rango entre 4 y 82 años. No se encontraron diferencias significativas relacionadas con la edad entre hombres (55.4 años) y mujeres (55 años). La localización más frecuente se evidenció en la glándula derecha (61.2\%) y se observó que a nivel global son más frecuentes las lesiones benignas $(58.9 \%)$ entre las cuales están el feocromocitoma $(15.3 \%)$, adenoma cortical $(12.8 \%)$, mielolipoma (7.6\%) e hiperplasia suprarrenal (5.1\%), no obstante, cabe resaltar que el diagnóstico con mayor frecuencia son las lesiones metastásicas (28.2\%). La descripción de las características demográficas se encuentra descrita en la Tabla 1. 
Tabla 1. Características demográficas de la población.

Lesión

\section{Sexo}

Hombres

Mujeres

Edad

Menores de 65

Mayores de 65

Localización

Derecha

Izquierda

Malignidad

No

Sí

Metástasis

No

Sí

Diagnóstico

Metástasis

Feocromocitoma

Adenoma cortical

Carcinoma Adrenocortical

Mielolipoma

Hiperplasia Suprarrenal

Paracoccidiodomicosis

Diagnóstico no claro

Necrosis

Hemorragia

Tumor Adenomatoide trombosado

Ganglioneuroma

\section{Largo}

Mayor a $4 \mathrm{~cm}$

Menor a $4 \mathrm{~cm}$

Fuente: elaboración propia.
Frecuencia $(n=39)$

48

29

29

10

80.5

19.5

62.16

37.84

14

23

16

58.97

41.03

21.43

78.57

11

28.2

6

15.3

5

4

3

10.2

7.6

2

2

2

1

1

1

1

70

7
90.9

5.1

5.1

2.5

2.5

2.5

2.5

2.5

9.09 
En cuanto a las características propias de las masas se encontró una media de largo de $8.15 \mathrm{~cm}$ (con un rango entre 2 - $23 \mathrm{~cm}$ ), la media del alto fue de $6.05 \mathrm{~cm}$ (con un rango entre $0.6-29 \mathrm{~cm}$ ), y la media del ancho encontrada fue de $3.9 \mathrm{~cm}$ (con un rango entre $0.5-10 \mathrm{~cm}$ ). La media del peso de las lesiones fue de $423.66 \mathrm{gr}$ (con un rango 11 - 2,025 gr). Estas características se resumen en la Tabla 2. También se observó que en los tumores de origen primario la gran mayoría son no malignos (82.1\%), y en aquellos tumores de origen metastásico el cáncer primario suele ser el cáncer renal de células claras (55.5\%), seguido del cáncer renal de ductos colectores (22.2\%); el resto de la distribución se representa en la Figura 1. En cuanto a la malignidad de acuerdo con el género, las masas malignas en mujeres fueron del $36.8 \%$ y en hombres representaron el $45 \%$; esta distribución se representa en la Figura 2.

Tabla 2. Características generales de las masas.

\begin{tabular}{ccc} 
Variable & Media & SD \\
\hline Largo $(\mathrm{cm})$ & 8.15 & 5.15 \\
\hline Alto $(\mathrm{cm})$ & 6.05 & 5.2 \\
\hline Ancho $(\mathrm{cm})$ & 3.9 & 2.43 \\
\hline Peso $(\mathrm{gr})$ & 423.66 & 2.43
\end{tabular}

Fuente: elaboración propia.

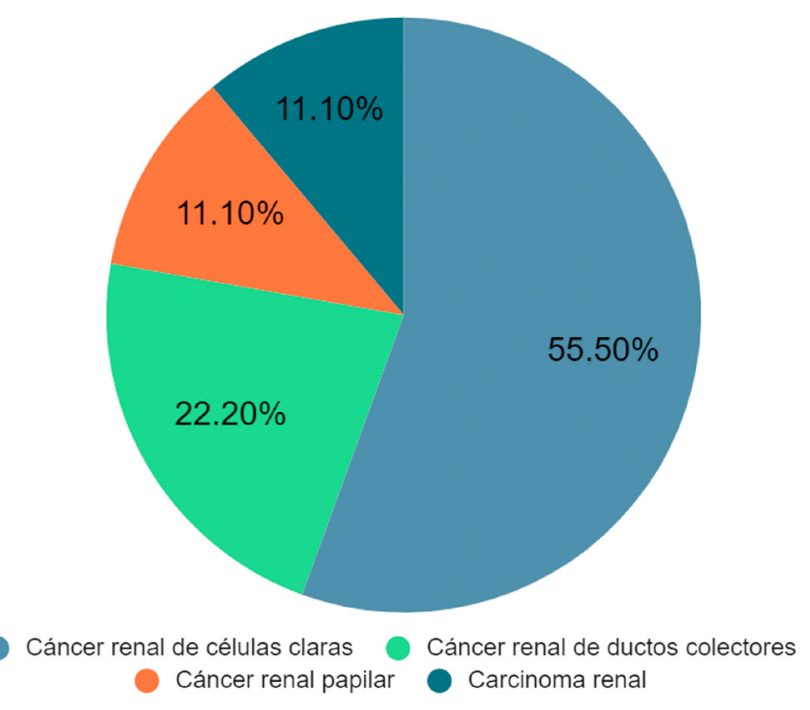

Figura 1. Distribución de los tumores de origen primario en las neoplasias de origen metastásico.

Fuente: elaboración propia.

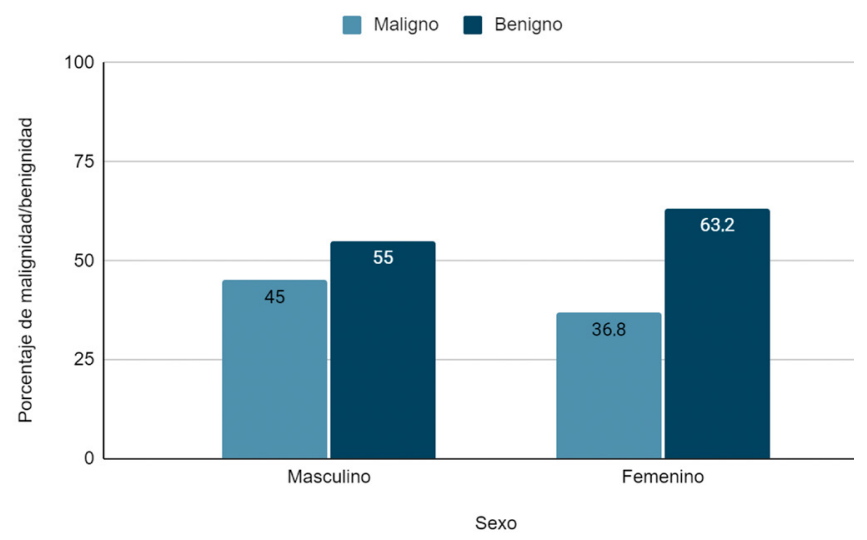

Figura 2. Relación entre la malignidad de las masas y el sexo.

Fuente: elaboración propia.

Al realizar el análisis bivariado, se buscaron asociaciones entre el tamaño de la masa ( $>4$ centímetros) y la malignidad de esta, sin hallar relaciones estadísticamente significativas $(\mathrm{p}=0.339)$. Al determinar la asociación entre edad y malignidad se encontró que los pacientes mayores a 65 años tienen asociación significativa de malignidad $(\mathrm{p}=0.04)$. Respecto a la asociación de la malignidad y el sexo no se encontraron diferencias estadísticamente significativas $(\mathrm{p}=0.650)$.

\section{Discusión}

En este estudio retrospectivo se evaluaron las características de un grupo de 39 patologías de la glándula suprarrenal, esto permite comparar los resultados obtenidos con la literatura internacional. Estas patologías fueron realizadas en pacientes con sospecha de cáncer, por ende, se consideran como masas de alto riesgo. Se encontraron similitudes en la epidemiología tales como la edad, en los estudios internacionales la incidencia aumenta con la edad, presentando un pico después de los 50 años, lo cual es coherente con el promedio de edad encontrado en el estudio que es de 55.2 años, esto también se encuentra en estudios realizados en Europa y en Argentina $(8,9)$. Adicionalmente, el estudio también corrobora que la glándula adrenal derecha es la más afectada y que es más frecuente la presencia de masa en hombres que en mujeres, aunque en el estudio no presentó asociaciones estadísticamente significativas, a nivel mundial sí hay estudios que lo demuestran $(13,14)$.

En cuanto a los diagnósticos histopatológicos, es importante resaltar la frecuencia significativa de las lesiones metastásicas encontradas en el estudio, así mismo, el origen primario del tumor, que en este caso 
la gran mayoría eran de origen renal, de células claras. Lo anterior es una diferencia relevante, ya que, a nivel internacional, el órgano con mayor frecuencia de metástasis a glándula adrenal en la mayoría de los estudios es el pulmón $(16,17)$. No obstante, también se ha descrito como primario principal el melanoma (18), cabe recalcar que las metástasis también pueden ser de mama, gástrico, renal, colon y páncreas $(17,19,20)$. En este estudio se presentan datos que permiten conocer la distribución y el comportamiento de las lesiones a nivel de la glándula suprarrenal en población colombiana, lo cual es útil en la práctica clínica para el diagnóstico diferencial y decisiones terapéuticas.

Adicionalmente, se pudo evaluar la necesidad de estandarización en cuanto a la las lecturas de las patologías, ya que en algunas de estas muestras faltaban datos fundamentales, desde la edad y el sexo del paciente hasta la descripción microscópica detallada y marcadores inmunohistoquimicos relevantes de cada patología, como por ejemplo lo hacen en un estudio de patologías urológicas desarrollado en Estados Unidos de América, donde unificaron criterios para la descripción de las muestras, lo cual resultaría útil en la práctica clínica (15). De igual forma, se requieren más estudios con datos de seguimiento prospectivo y que integren variantes clínicas para un conocimiento más profundo de la patología a nivel de la glándula suprarrenal propio de esta región.

\section{Conclusión}

Al realizar la descripción de los hallazgos histopatológicos y la malignidad de las glándulas suprarrenales se encontró que las variantes como la edad, el sexo y la lateralidad de afectación de la glándula adrenal, tienen frecuencias similares con la epidemiología descrita a nivel mundial. A pesar de que no se encontraron asociaciones estadísticamente significativas, se considera necesario nuevos estudios con seguimiento prospectivo y que integren datos clínicos para comprender mejor el comportamiento de estas lesiones y, a su vez, continuar con la generación de epidemiología local que permite mejorar el diagnóstico, tratamiento y pronóstico de las mismas.

\section{Declaración de fuentes de financiación y posibles conflictos de interés}

No requirió financiación.

No presentamos conflictos de interés.

\section{Consideraciones éticas}

Este es un estudio observacional descriptivo, conducido bajo los criterios y parámetros establecidos por la Resolución 8430 de 1990. El estudio utilizó datos de registro médico e histopatológico recolectados en una institución de Bucaramanga entre 2007 y 2019, y cuenta con el aval del Comité Técnico.

\section{Referencias}

1. Betancourt VD, Archila EI. Tumores suprarrenales. Revisión bibliográfica. Acta Méd Centro [Internet]. 2014 [citado 17 Mar 2021]; 8(1):[aprox. 10 p.]. Disponible en: http://www.revactamedicacentro.sld. cu/index.php/amc/article/view/59/158

2. Arnaldi G, Masini A.M, Giacchetti G, Taccaliti A, Faloia E, Mantero F. Adrenal incidentaloma. Braz J Med Biol Res [Internet]. 2000 Oct [cited 2021 Mar 17]; 33(10): 1177-1189. https://doi.org/10.1590/ S0100-879X2000001000007

3. Mannelli M, Colagrande S, Valeri A, Parenti G. Incidental and metastatic adrenal masses. Semin Oncol. 2010;37(6):649-61. https://doi.org/10.1053/j. seminoncol.2010.10.018

4. Long SE, Miller BS. Adrenocortical cancer treatment. Surg Clin North Am. 2019;99(4):759-71. https://doi. org/10.1016/j.suc.2019.04.012

5. Kloss RT, Gross MD, Francis IR, Korobkin M, Shapiro B. Incidentally discovered adrenal masses. Endocr Rev. 1995 Aug;16(4):460-84. https://doi. org/10.1210/edrv-16-4-460

6. Oliveira R, Salvador R, Buñesch L, Sebastià MC, Nicolau C. Manejo y diagnóstico del incidentaloma suprarrenal. Radiología. 2011;53(6):516-30. https:// doi.org/10.1016/j.rx.2011.06.006

7. Román-González A, Agredo-Delgado V, AristizábalBarón J, Arizmendy-Acosta D. Prevalencia de mielolipoma como diagnóstico incidental de masas adrenales en tomografias abdominales realizadas en el Hospital Universitario San Vicente Fundación de Medellín. Iatreia. 2018 Dec [cited 2021 Mar 17]; 31(4): 342-350. Disponible en: http://www.scielo.org.co/scielo.php? script $=$ sci arttext\&pid=S0121-07932018000400342

8. Gómez RM, Chervín R, Pardes EM, Lupi S, Surraco $\mathrm{ME}$, Herrera $\mathrm{J}$, et al. Evaluación diagnóstica $\mathrm{y}$ terapéutica del incidentaloma suprarrenal. Rev Argent Endocrinol Metab. 2016;53(2):51-8. https://doi. org/10.1016/i.raem.2015.12.001

9. Fassnacht M, Arlt W, Bancos I, Dralle H, NewellPrice J, Sahdev A et al. Management of adrenal incidentalomas: European Society of Endocrinology 
Clinical Practice Guideline in collaboration with the European Network for the Study of Adrenal Tumors. European journal of endocrinology. 2016 Aug;175(2):G1-G34. https://doi.org/10.1530/EJE16-0467

10. Hevia M, Abascal JM, Boix P, Dieguez M, Delgado E, Abascal JM. et al. Manejo de la masa suprarrenal: lo que el urólogo debe saber. Actas Urol Esp. 2010 Ago [citado 2021 Mar 17]; 34(7): 586-591. https:// doi.org/10.1016/j.acuro.2009.11.010

11. Vaidya A, Hamrahian A, Bancos I, Fleseriu M, Ghayee HK. The evaluation of incidentally discovered adrenal masses. Endocr Pract. 2019;25(2):178-92. https://doi. org/10.4158/DSCR-2018-0565

12. Ferreira E, Czepielewski M, Faccin C, Coral M, Furtado A.Prevalência de lesão adrenal incidental em pacientes submetidos a tomografia computadorizada de tórax e abdome em um hospital geral brasileiro. Arq Bras Endocrinol Metab. 2005 Out [citado 2021 Mar 17]; 49(5): 769-775. https://doi.org/10.1590/ S0004-27302005000500017

13. Ctvrtlik F, Koranda P, Tichy T. Adrenal disease: a clinical update and overview of imaging. A review. Biomed Pap Med Fac Univ Palacky Olomouc Czech Repub. 2014;158(1):23-34. https://doi.org/10.5507/ bp. 2014.010

14. Domínguez LE, Marín C, Díaz M, Jiménez A. Incidentaloma Suprarrenal: estado de la cuestión. Cir Andal [Internet]. 2014 Marzo; 25(1):53-9. Disponible en: https://www.asacirujanos.com/admin/upfiles/ revista/2014/2014-vol25-n1-act6.pdf
15. Hansel DE, Reuter VE. Adrenal Pathology in the Adult: A Urological Pathologist's Perspective. Advances in anat. 2016 sep;23(5):273-84. https://doi. org/10.1097/PAP.0000000000000120

16. De la Quintana A, Martínez G, Arana A, Prieto M, Álvarez I, Martínez L, et al. Cirugía de las metástasis en la glándula suprarrenal: resultados de una serie de 35 pacientes. Cir Esp. 2012;90(10):634-40. https:// doi.org/10.1016/j.ciresp.2012.04.013

17. Alshahrani MA, Bin Saeedan M, Alkhunaizan T, Aljohani IM, Azzumeea FM. Bilateral adrenal abnormalities: imaging review of different entities. Abdom Radiol (NY). 2019;44(1):154-79. https://doi. org/10.1007/s00261-018-1670-5

18. Angelousi A, Alexandraki KI, Kyriakopoulos G, Tsoli M, Thomas D, Kaltsas G, et al. Neoplastic metastases to the endocrine glands. Endocr Relat Cancer. 2020;27(1):R1-20. https://doi.org/10.1530/ERC-19$\underline{0263}$

19. Gupta P, Bhalla A, Sharma R. Bilateral adrenal lesions: Bilateral. J Med Imaging Radiat Oncol. 2012;56(6):636-45. https://doi.org/10.1111/j.17549485.2012.02435.x

20. Wagnerova H, Lazurova I, Felsoci M. Adrenal metastases. Bratisl Lek Listy. 2013;114(4):237-40. https://doi.org/10.4149/BLL_2013 049 